\title{
ELABORATION AND GRAFTING OF MAGNETIC BEAD-CHAINS FOR DETECTION OF ANISOTROPY WITH POLARIMETRIC SURFACE PLASMON RESONANCE IMAGING SYSTEM
}

\author{
M. TREVISAN, Y. CHEVOLOT, V. MONNIER, J. P. CLOAREC* \\ and E. SOUTEYRAND \\ Université de Lyon \\ Institut des Nanotechnologies de Lyon, UMR CNRS 5270- site \\ Ecole Centrale de Lyon \\ 36, Avenue Guy de Collongue, 69130 Ecully, France \\ *jcloarec@ec-lyon.fr \\ A. DUVAL, J. MOREAU and M. CANVA ${ }^{\dagger}$ \\ Laboratoire Charles Fabry \\ Institut d'Optique Graduate School, CNRS \\ Université Paris Sud, Campus Polytechnique - 2 \\ Avenue Augustin Fresnel - 91127-Palaiseau, France \\ ${ }^{\dagger}$ michael.canva@institutoptique.fr \\ Received 1 December 2011 \\ Accepted 2 May 2012 \\ Published 10 August 2012
}

\begin{abstract}
The aim of this report is to prove that polarimetric surface plasmon resonance imaging (P-SPRI) is able to characterize dynamically the anisotropy of micro and nanoobjects. Micro and nanoparticles were assembled into filaments at a specific location on a support, using a combination of magnetic field, amine based chemistry and orthogonal surface chemistry. After immobilization of the filaments onto gold pads, they were actuated by a magnetic field and validated the P-SPRI system.
\end{abstract}

Keywords: Surface chemistry; SPR; anisotropy; magnetic bead filaments.

\section{Introduction}

Over the last decade, the interest for elaboration of nanoobjects has strongly increased. Among various ways for building customized nanoobjects, the bottom-up approach (from molecules to the object) seems promising although some difficulties such as the precise collective location of molecules onto support. In parallel, the characterization and the study of behavior of such objects required new tools adapted to the submicro or nanoscale. Here, we report a methodology based on the use of magnetic particles and their manipulation by a magnetic field 
to self-assemble them into permanent filaments ${ }^{1,2}$ thanks to amine based chemistry. These permanent magnetic filaments made from micro $(1 \mu \mathrm{m})$ or nano $(200 \mathrm{~nm})$ beads have been collectively addressed onto support on specific area thanks to a selective chemical functionalization. ${ }^{3}$ Then, the filaments could be actuated by an external magnetic field while keeping their stable anchorage on the surface: Their orientation is tunable in real time within a $2 \pi$ solid angle. Thus, they have been used to prove that a polarimetric surface plasmon resonance imaging $(\mathrm{P}-\mathrm{SPRI})^{4,5}$ system is sensitive to the change of anisotropy of submicro or nanoobjects performed in situ in liquid media.

\section{Experimental}

\subsection{Polarimetric surface plasmon resonance imaging system (P-SPRI)}

P-SPRI system is constituted of two independent SPRI sensing arms based on Kretschmann-Raether configuration and disposed orthogonally to each other. ${ }^{1}$ At the same time and at the same location, the plasmon waves resulting from each arm's orientation probe the biological surface along two orthogonal directions. The P-SPRi system has been characterized $^{2}$ and performances are reported in Table 1.

To prevent a potential sensitivity difference between the two arms susceptible to generate a false anisotropy, an intra and inter arms normalization is required. Thus, a measurement protocol in five steps has been established including:

(1) Optimal angle determination

(2) For a fixed angle, sensitivity $(\Delta R i / \Delta n)$ determination at the point $i$ of the sample for each arm

(3) Real time signal measurement for each arm

(4) Determination of displacement vector allowing to cancel the shift between the 2 arms $x$ and $y$.

(5) Data post treatment and measurement of anisotropy through reflectivity difference $\Delta R=$ $\Delta R_{x}-\Delta R_{y}$

A microscope equipped with a CDD camera and a white light source were also located above the SPR set-up. A chopper enabled to record in real time alternatively P-SPR signals and upper face of SPR supports optical pictures.
Table 1. Performances of P-SPRI systems.

\begin{tabular}{ll}
\hline Wavelength $\lambda$ & $670 \mathrm{~nm}$ \\
Angular range $\theta$ & $47^{\circ}-60^{\circ}$ \\
Refractive index range & $1.29-1.39$ \\
Maximum Materials & $50 \mathrm{~nm}$ \\
$\quad$ Thickness $(n=1.48)$ & \\
Measurable anisotropy & $310^{-4} \mathrm{RIU}$ \\
Measure Noise & $10^{-2} \%(50 \times 50$ pixels $) / 0.31 \% /$ \\
Signal to Noise Ratio & $46,4 \mathrm{~dB}$ \\
\hline
\end{tabular}

\subsection{Localization and actuation of permanent filaments onto mixed support}

A simple method combining magnetic field and microparticles has been designed by our team for assembling, locating and grafting permanent microfilaments onto homogeneous support. ${ }^{3}$ Formation and location of filaments were monitored by using magnetic tips set under the support. Here, we used a mixed support constituted of gold square areas $\left(1 \times 1 \mathrm{~mm}^{2}\right)$ spaced of $0.5 \mathrm{~mm}$ onto glass slide (SF11). Thickness of homogeneous gold layer on squares was $47 \mathrm{~nm}$ with a $\mathrm{Cr}$ under layer of $3 \mathrm{~nm}$.

\subsubsection{Surface preparation}

A selective double functionalization has been achieved as presented in Fig. 1. First step consisted in dipping sample into a 11-mercaptoundecanoic acid solution ( $2 \mathrm{mM}$ in isopropanol) at $4^{\circ} \mathrm{C}$ for $48 \mathrm{~h}$ to form a self assembled monolayer (SAM) of Alkylthiols. As alkylthiols have a stronger affinity with metals than glass (and more particularly with

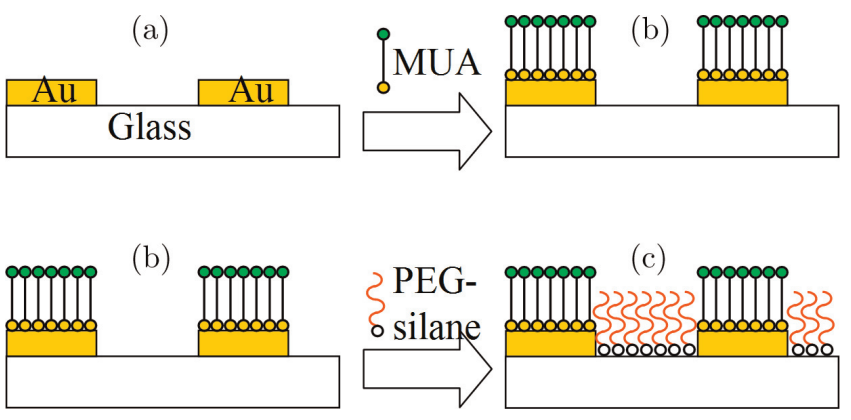

Fig. 1. Selective surface functionalization made in two steps onto mixed $\mathrm{Au} /$ glass support. Mercapto-undecanoic acid forms a monolayer on gold, while PolyEthylene Glycol silane reacts with glass. 
gold), the following washing step with isopropanol (30 min ultrasounic bath) allowed removing them from glass surface while maintaining the chemical functionalization of the gold areas.

A second step aimed the glass surface silanization by immersion into a PEG-silane (PolyEthyleneGlycol) solution (215 $\mu \mathrm{l}$ in $50 \mathrm{ml}$ of dichloromethane) for $48 \mathrm{~h}$ at room temperature. Samples were rinsed successively in dichloromethane, in $0.1 \%$ SDS (Sodium Dodecyl Sulfate) and finally in ultrapure water for $10 \mathrm{~min}$ under ultrasonic bath. Before their use, samples have to be activated overnight at room temperature with $125 \mathrm{ml}$ of tetrahydrofurane (THF), $1.5 \mathrm{~g}$ of N-Hydro Succinimide (NHS) and $2 \mathrm{ml}$ of N,N'-diisopropyl carbodiimide (DCI) solution then washed in THF and finally in dichloromethane for $10 \mathrm{~min}$.

\subsubsection{Assembling, locating and grafting permanent microfilaments onto gold surface}

Magnetic carboxylatex particles of $1 \mu \mathrm{m}$ diameter (provided by Sigma) were first activated with EDCI (1-3-(Dimethylamino) propyl)-3-ethylcarbodiimide methiodide)/NHS for $2 \mathrm{~h}$ at room temperature under intermittent agitation, rinsed three times in SDS $0.1 \%$ solution and then dispersed in controlled volume solution to determine their concentration before to be deposited in solution onto an activated solid support. By applying a magnetic field orthogonal to the surface, the magnetic particles selfassembled in chains. PEG-diamine chains were then used to covalently bind two particles or a particle and the support ${ }^{2}$ (Fig. 2). Supports were washed with aqueous SDS $0.1 \%$ to remove excess particles and nonanchored filaments.

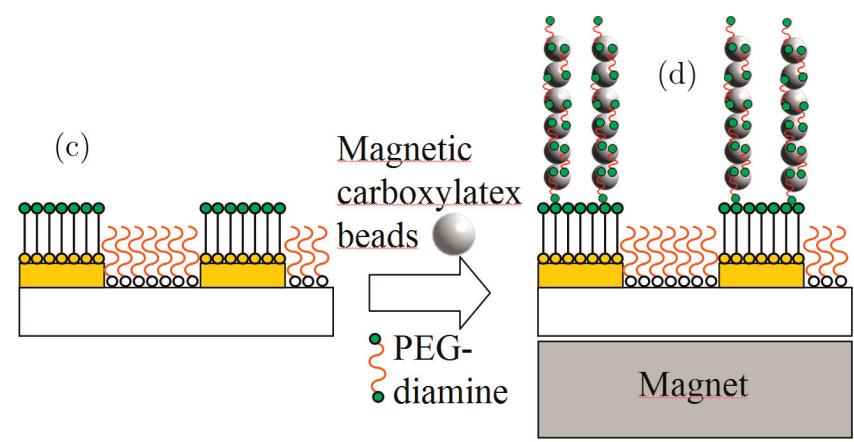

Fig. 2. Selective grafting of magnetic beads made filaments only onto Au spot of support. This step follows steps of Fig. 1.

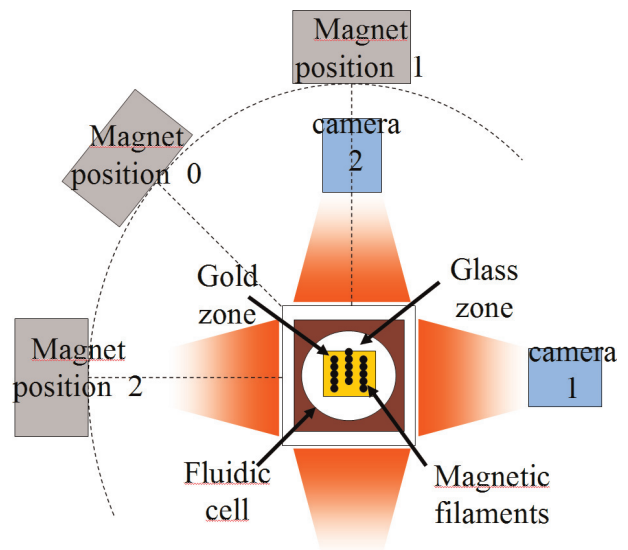

Fig. 3. Magnet position used for filament actuation during the experiments for the validation of P-SPRI sensor. Pos0, Pos1 and Pos 2 correspond respectively to $45^{\circ}, 0^{\circ}$ and $90^{\circ}$ compared to the vertical axis.

A similar methodology was adapted to $200 \mathrm{~nm}$ diameter magnetic beads provided by Ademtech. These filaments were grafted covalently on the gold part of a mixed support thanks to the selective functionalization and could also be actuated.

\subsubsection{Real time monitoring of actuated filaments}

Supports bearing magnetic filaments immobilized onto gold zones were covered with a fluidic chamber, immersed in water, in order to keep filaments in a liquid phase and avoid any drying.

Supports were analyzed with the SPR/optical microscopy set-up. During measurements filaments were actuated using a $0.3 \mathrm{~T}$ Neodyme magnet, positioned at $5 \mathrm{~cm}$ from the center of the sample.

Magnet was positioned at three different locations (Fig. 3), in order to actuate the immobilized filaments in three different orientations.

\section{Results and Discussion}

\subsection{Filaments assembly}

Filaments made of $1 \mu \mathrm{m}$ and $200 \mathrm{~nm}$ diameter beads were observed by optical microscopy (data not shown) and scanning electronic microscopy (SEM) (Fig. 4). Optical microscopy enabled observation of $1 \mu \mathrm{m}$ bead filaments in liquid medium. Filaments made of $200 \mathrm{~nm}$ magnetic beads could not be observed with optical microscopy and were characterized by SEM. After self assembly, the filaments 


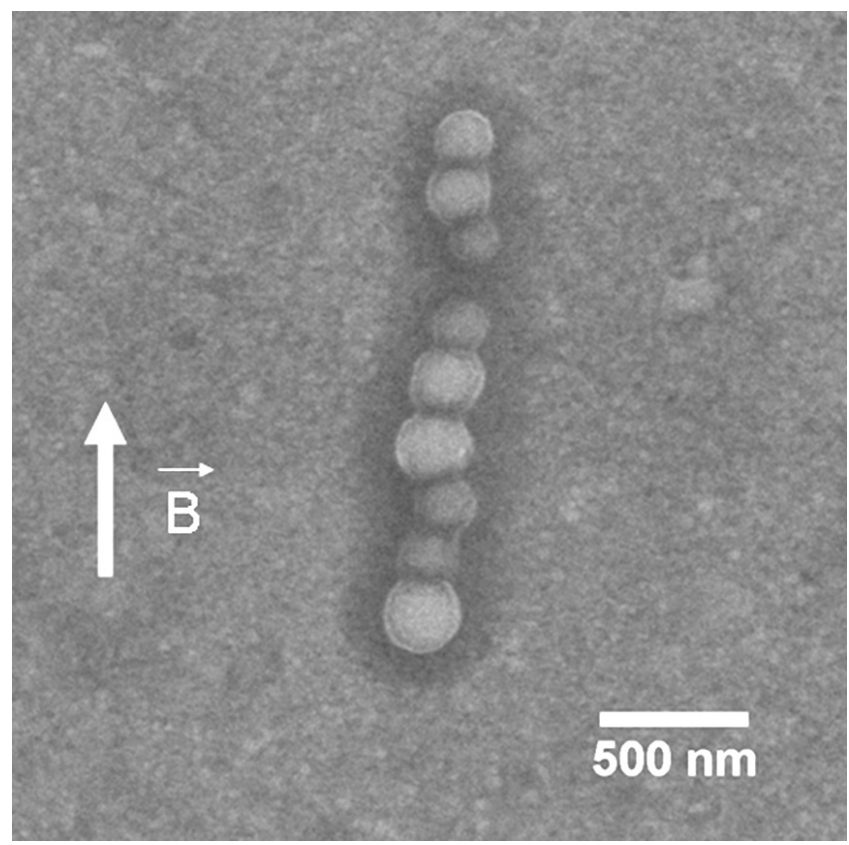

Fig. 4. SEM observation of isolated filament made of $200 \mathrm{~nm}$ diameter magnetic beads surrounded with a jeffamine shell. The arrow indicates the direction of the magnetic field during drying of the sample.

were run dry under magnetic field, in order to absorb all the filaments in a same direction. After drying, filaments kept the same orientation, probably due to adsorption onto the support. They could be analyzed with SEM.

\subsection{Selective immobilization of filaments onto gold}

After chemical anchoring of the $1 \mu \mathrm{m}$ beads filaments and washing, filaments could be observed by optical microscopy only on gold zones, while no visible filament were detected onto glass zones (Fig. 5). This suggests that the selective chemical functionalization of gold $(\mathrm{COOH})$ and glass $(\mathrm{PEG})$ was effective in addressing and grafting of filaments on carboxylated modified gold, and limited adsorption of filaments on PEG-silanized glass.

\subsection{P-SPR monitoring of actuated filaments}

The anisotropy is characterized by a reflectivity difference $(\Delta R)$ between the two orthogonal arms of the SPR sensor. For all measurements, all the magnetic filaments were oriented parallel to the gold surface. The change of magnet position only

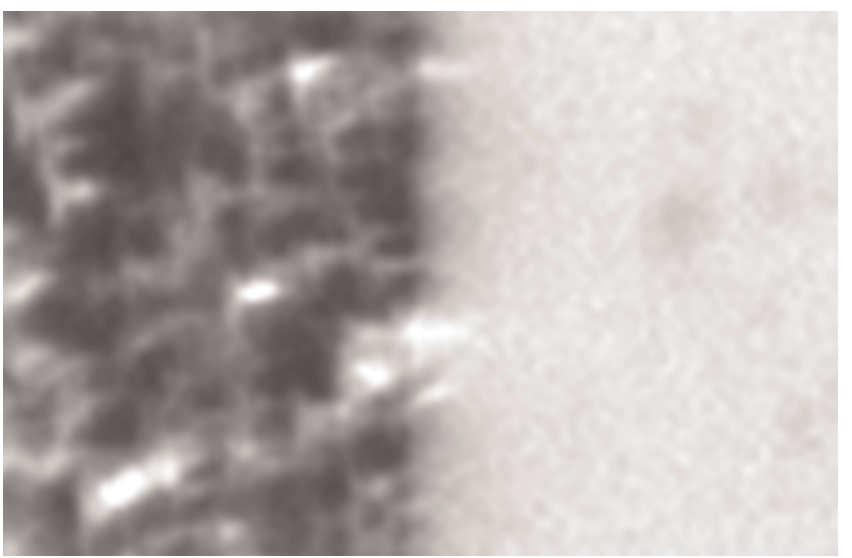

Fig. 5. (Color online) Optical microscope image of Gold/glass support after grafting of filaments. Left: gold zone covered with filaments (dark aspect). Right: glass zone. The few dark spots on glass are attributed to optics, and are not due to filaments.

affected the angle of the filaments, while keeping filaments parallel to the gold surface, in the horizontal plane. This enabled to keep constant the mass of filaments probed by SPR analysis, while probing the plasmon propagation in the two directions of the axis 1 and 2. In Fig. 6, $\Delta R$ corresponds to the difference of reflectivities between camera 1 and camera 2, for a same geometric point of the analyzed support. This measurement thus characterizes the difference of SPR behavior, depending on the anisotropy of the surface. $\Delta R$ was measured for a reference spot (gold without

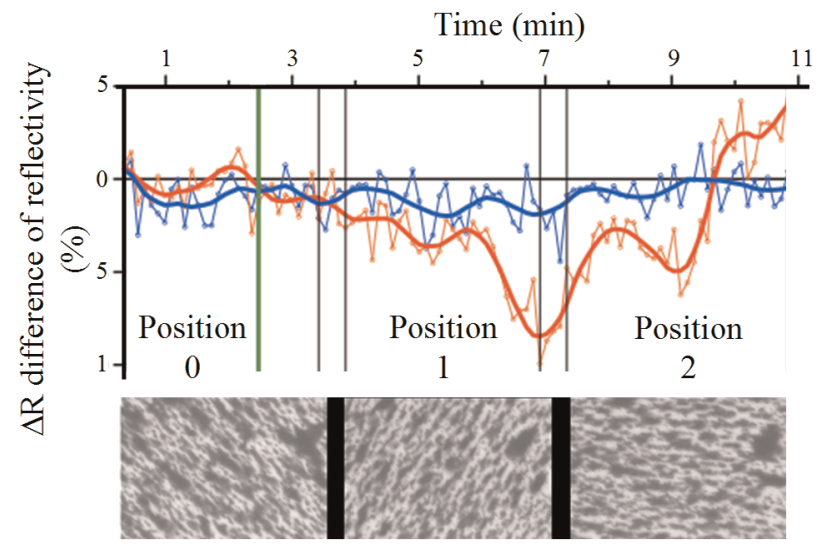

Corresponding in situ optical microscopy image

Fig. 6. (Color online) (top) real time measurement of reflectivity difference with a polarimetric Surface Plasmon Resonance Imaging sensor during the actuation of micronic permanent filaments on reference spot (blue line) and spot with filament (red line). (bottom) Corresponding real-time filament imaging by optical microscopy. 
filaments: Fig. 6 blue curve) and a spot with filaments (Fig. 6 red curve).

Abrupt signal variations were observed when the magnet position and so the filament angle was changed compared to axis 1. $\Delta R$ changed from a value of $-0.01 \%$ at $45^{\circ}$ to a value of $-0.08 \%$ at $0^{\circ}$. This phenomenon was observed between $0^{\circ}$ and $90^{\circ}$ : From $\Delta R=-0.08 \%$ to $\Delta R=-0.04 \%$. Weak signals are probably due both to the low coverage of filaments onto surface which induces low change in anisotropy and also to the use of $1 \mu \mathrm{m}$ diameter beads. In our conditions, surface plasmons penetrated only on the first $200 \mathrm{~nm}$ above the surface which means that less than $40 \%$ of the beads volume at the surface is being probed.

Thus, we can conclude that first measurements tend to show that the filament anisotropy can be detected by the P-SPRI biosensor. One way to improve the signals would be to use smaller beads $(200 \mathrm{~nm})$ but in this case, it will be necessary to increase the concentration of bead - chains grafted onto surface and also to well monitor their actuation to leave out the simultaneous microscopic observations.

\section{Acknowledgments}

The authors acknowledge the financial support of National Research Agency (ANR) through the Program for Nanosciences and Nanotechnologies (PNANO) and the European Network of Excellence Photonics4Life.

\section{References}

1. C. Goubault, F. Leal-Calderon, J. L. Viovy and J. Bibette, Langmuir 21, 3725 (2005).

2. N. Haddour, Y. Chevolot, M. Trevisan, E. Souteyrand and J.-P. Cloarec, J. Mater. Chem. 20, 8266 (2010).

3. E. Briand, V. Humblot, J. Landoulsi, S. Petronis, C.-M. Pradier, B. Kasemo et al., Langmuir 27, 678 (2011).

4. F. Bardin, A. Bellemain, G. I. Roger and M. Canva, Biosens. Bioelectron. 24, 2100 (2009).

5. A. Duval, A. Laisné, D. Pompon, S. Held, A. Bellemain, J. Moreau et al., Opt. Lett. 34, 3634 (2009). 